The Astrophysical Journal, 677:572-580, 2008 April 10

(C) 2008. The American Astronomical Society. All rights reserved. Printed in U.S.A.

\title{
COULD THE ULTRA-METAL-POOR STARS BE CHEMICALLY PECULIAR AND NOT RELATED TO THE FIRST STARS?
}

\author{
K. A. VENN \\ Department of Physics and Astronomy, University of Victoria, Elliott Building, 3800 Finnerty Road, \\ Victoria, BC V8P 5C2, Canada; kvenn@uvic.ca \\ AND \\ DAVID L. LAMBeRT \\ W. J. McDonald Observatory, University of Texas at Austin, RLM 15.308, Austin, TX 78712; d11@astro.as.utexas.edu \\ Received 2007 October 19; accepted 2008 January 1
}

\begin{abstract}
Chemically peculiar stars define a class of stars that show unusual elemental abundances due to stellar photospheric effects and not due to natal variations. In this paper, we compare the elemental abundance patterns of the ultra-metalpoor stars with metallicities $[\mathrm{Fe} / \mathrm{H}] \sim-5$ to those of a subclass of chemically peculiar stars. These include post-AGB stars, RV Tauri variable stars, and the Lambda Bootis stars, which range in mass, age, binarity, and evolutionary status, yet can have iron abundance determinations as low as $[\mathrm{Fe} / \mathrm{H}] \sim-5$. These chemical peculiarities are interpreted as due to the separation of gas and dust beyond the stellar surface, followed by the accretion of dust-depleted gas. Contrary to this, the elemental abundances in the ultra-metal-poor stars are thought to represent yields of the most metal-poor supernovae and, therefore, observationally constrain the earliest stages of chemical evolution in the universe. Detailed chemical abundances are now available for HE 1327-2326 and HE 0107-5240, the two extreme ultra-metal-poor stars in our Galaxy, and for HE 0557-4840, another ultra-metal-poor star found by the Hamburg/ESO survey. There are interesting similarities in their abundance ratios to those of the chemically peculiar stars; e.g., the abundances of the elements in their photospheres are related to the condensation temperature of that element. If these three stars are chemically peculiar, then their $\mathrm{CNO}$ abundances suggest true metallicities of $[\mathrm{X} / \mathrm{H}] \sim-2$ to -4 . It is important to establish the nature of these stars, since they are used as tests of the early chemical evolution of the Galaxy.
\end{abstract}

Subject headings: early universe — stars: abundances — stars: chemically peculiar -

stars: individual (HE 1327-2326)

\section{INTRODUCTION}

A fossil record of the earliest episodes of stellar nucleosynthesis in the universe and Galaxy should be revealed by the compositions of the most metal-poor Galactic stars (e.g., Tumlinson 2007a, 2007b; Tominaga et al. 2007; Umeda \& Nomoto 2003; Suda et al. 2004). The lure of this revelation has driven the search to find and analyze such Rosetta stones. A great leap forward was achieved recently by the discovery of two stars with iron abundances $[\mathrm{Fe} / \mathrm{H}]<-5.3$ (Christlieb et al. 2002; Frebel et al. 2005), a limit about 1.5 dex below the abundance of the previously known most metal-poor star. A third star with $[\mathrm{Fe} / \mathrm{H}] \simeq-4.8$ also beats the previous lower bound (Norris et al. 2007). Prior to these remarkable discoveries, the most $\mathrm{Fe}$-poor stars known were HR 4049 and HD 52961 with $[\mathrm{Fe} / \mathrm{H}] \simeq-4.8$ (e.g., Waelkens et al. 1991). However, these and slightly more iron-rich examples were dismissed - correctly - as irrelevant to the issue of early stellar nucleosynthesis because they are "chemically peculiar"; i.e., their present surface compositions are far removed from their initial compositions. In particular, their compositions reflect that of gas from which refractory elements have been removed to varying degrees by a process dubbed "dust-gas separation." The existence of HR 4049 and HD 52961 has led us to reexamine the question of whether the recently discovered ultra-metal-poor stars may themselves be chemically peculiar.

Two of the three ultra-metal-poor stars in question are HE13272326 and HE $0107-5240$. HE 1327-2326 was discovered by Frebel et al. (2005), and abundance analyses have been described by Aoki et al. (2006), Frebel et al. (2006), Collet et al. (2006), and most recently by A. Frebel \& N. Christlieb (2007, private com- munication). The latter analysis based on the highest $\mathrm{S} / \mathrm{N}$ spectra yields abundances for 11 elements and upper limits for an additional nine elements: the new iron abundance at $[\mathrm{Fe} / \mathrm{H}]=-5.9$ is even lower than the previous determination. HE 0107-5240 was discovered by Christlieb et al. (2002), and abundance analyses have been reported by Christlieb et al. (2004), Bessell et al. (2004), and Collet et al. (2006) with the latter suggesting $[\mathrm{Fe} / \mathrm{H}] \simeq$ -5.6 . The third star, HE $0557-4840$, was discovered and analyzed by Norris et al. (2007): $[\mathrm{Fe} / \mathrm{H}]=-4.8$ places it between the two ultra-metal-poor stars (HE 1327-2326 and HE 0107-5240) and the lower boundary of the metal-poor tail of Galactic stars.

A marked characteristic of these three discoveries is that some abundance ratios are uncharacteristic of metal-poor stars of higher $\mathrm{Fe}$ abundance. Notably, the stars are C-rich for their Fe abundance, i.e., $[\mathrm{C} / \mathrm{Fe}]=3.7$ for $\mathrm{HE} 1327-2326$, and also $[\mathrm{N} / \mathrm{Fe}]=$ 4.1 and $[\mathrm{O} / \mathrm{Fe}]=3.4$ (A. Frebel \& N. Christlieb 2007, private communication). This property of unusual abundance ratios is shared in a qualitative sense with HR 4049 and HD 52961.

In the following sections, we briefly review the classes of known chemically peculiar stars affected by dust-gas separation. Then, we discuss whether the three stars HE 1327-2326, HE 0107-5240, and HE $0557-4840$ are chemically peculiar rather than true ultrametal-poor stars. In the final sections, we discuss possible tests of the hypothesis that ultra-metal-poor stars may be chemically peculiar.

\section{SEPARATION OF GAS AND DUST AND CHEMICALLY PECULIAR STARS}

The chemically peculiar stars in question are those whose atmospheres betray the operation of the dust-gas separation process. 


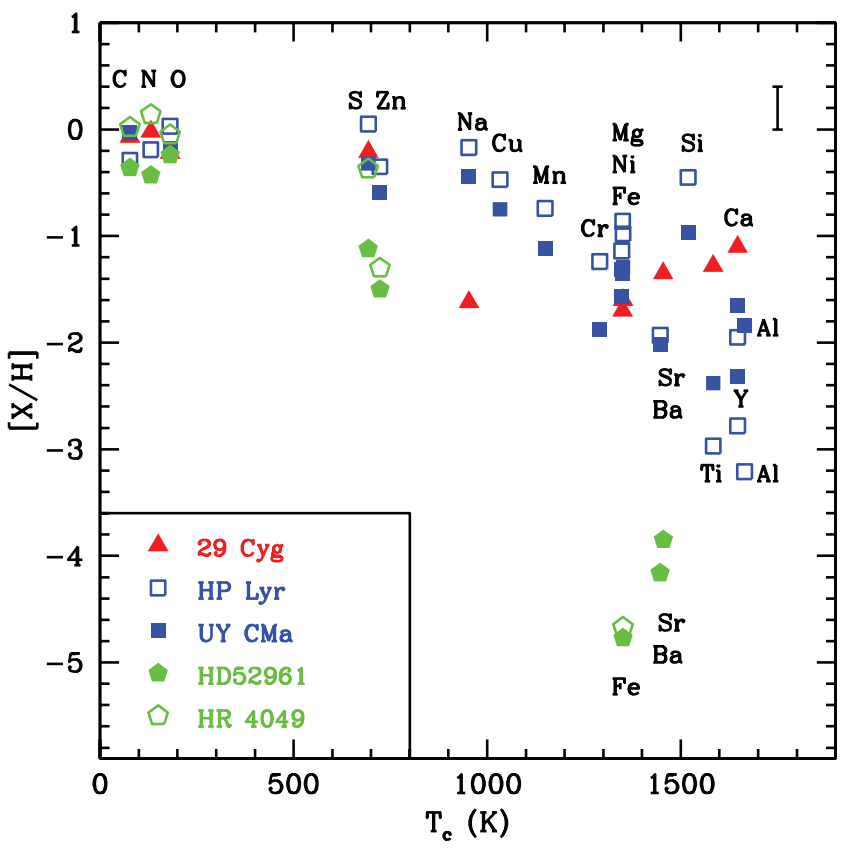

FIG. 1.-Elemental abundances of chemically peculiar stars relative to solar; including the Lambda Bootis star, 29 Cyg (red; Venn \& Lambert 1990), two RV Tauri stars, UY CMa and HP Lyr (Giridhar et al. 2005), and two A-type post-AGB stars, HR 4049 and HD 52961 (Waelkens et al. 1991, with Zn from Takeda et al. 2002 and van Winckel et al. 1992). Elemental abundances are plotted against the solar condensation temperatures from Lodders (2003). Typical errors are $\leq 0.2 \mathrm{dex}$, shown by the error bar in the upper right.

In gas of a sufficiently low temperature, dust condenses out and the gas is depleted in those elements that form the dust. The local interstellar gas, for example, displays such depletion (Savage \& Sembach 1996). The composition of the gas in a dust-gas mixture depends on several primary factors including the initial composition of the gas, the total pressure, and the history of the gas-dust mixture. If a star were to then accrete gas, preferentially over dust, and the accreted gas were to comprise a major fraction of the stellar photosphere, a star with striking abundance anomalies results. This scenario is one version of how a star could develop chemical peculiarities from dust-gas separation (or "dustgas winnowing"). Anomalies plausibly attributable to dust-gas separation have now been reported for three kinds of stars: Lambda Bootis stars, post-AGB A-type spectroscopic binaries, and RV Tauri variables.

The Lambda Bootis stars are main-sequence stars, ranging from A to mid-F types, and found at all evolutionary phases from very young (e.g., they are found in young open clusters; Gray \& Corbally 1998) to the end of the main sequence (Kamp \& Paunzen 2002; Andrievsky et al. 2002). They are expected to have a solarlike composition but have long been known for their significant metal deficiencies (Morgan et al. 1943; Burbidge \& Burbidge 1956; Baschek \& Searle 1969). These stars have effective temperatures of from about 6500 to $9500 \mathrm{~K}$ and surface gravities $\log g \simeq 4$. Our (Venn \& Lambert 1990) abundance analysis of three stars, including the eponym, showed that the compositions of the stars could be attributed to accretion of circumstellar gas, without dust. The iron deficiency in the case of $\lambda$ Boo was $[\mathrm{Fe} / \mathrm{H}] \simeq-2$, and slightly less severe for $29 \mathrm{Cyg}$, with normal abundances of $\mathrm{C}, \mathrm{N}, \mathrm{O}$, and $\mathrm{S}$ for both stars, in agreement with the expectations for an atmosphere contaminated with dust-free gas (see Fig. 1). Even Vega, a "standard" A0 star, was shown to be a mild Lambda Bootis star (Venn \& Lambert 1990; Lemke \& Venn 1996). Vega also shows an infrared excess due to a dusty circumstellar disk ( $\mathrm{Su}$ et al. 2005; Aumann et al. 1984).

The shallow convective envelope of early A-type stars is a key factor in the creation and maintenance of the abundance anomalies in the diffusion/accretion model (Turcotte \& Charbonneau 1993). Abundance anomalies can persist $\left(\sim 10^{6} \mathrm{yr}\right)$, even after dispersal of the circumstellar dust and gas and, hence, removal of the infrared excess contributed by the dust. Thus, not all Lambda Bootis stars show an infrared excess; Paunzen et al. (2003) estimate that $23 \%$ of bona fide Lambda Bootis stars show evidence for circumstellar matter. On the other hand, the circumstellar matter may be of interstellar origin (Kamp \& Paunzen 2002; Gáspár et al. 2008). Movement of a star through the denser parts of the interstellar medium can create a bow shock, which heats the interstellar dust causing an infrared excess. Meanwhile, radiation pressure from the star repels the grains, while gas is accreted onto the stellar surface. With the return of the star to passage through lowdensity gas, the infrared excess dissipates and accretion ceases. This alternate theory for the origin of the Lambda Bootis stars implies that the chemical anomalies are transient but could help to explain why the phenomenon is seen in such a wide range of main-sequence stars.

Abundance anomalies should not survive the transition from the main sequence to the giant branch, however. The deep convective envelope of giant stars will surely dilute abundance anomalies beyond recognition. Yet, a metal deficiency of even greater severity can be found among post-AGB stars in spectroscopic binaries (Waelkens et al. 1991; van Winckel 2003). These stars, like HR 4049 and HD 52961 discussed above, are supergiants with $T_{\text {eff }}$ in the range of $6000-7600 \mathrm{~K}$ and surface gravities $\log g \simeq 1$. One must suppose that a new episode of dust-gas separation led to these abundance anomalies.

The original quartet of post-AGB binaries (van Winckel et al. 1995) comprised HR 4049, HD 44179, HD 52961, and BD +39 4926. Their $[\mathrm{Fe} / \mathrm{H}]$ values range from -3.0 to -4.8 , but they show an abundance pattern reminiscent of interstellar gas, i.e., quasi-solar abundances of $\mathrm{C}, \mathrm{N}, \mathrm{O}, \mathrm{S}$, and $\mathrm{Zn}$, but severe underabundances of, for example, $\mathrm{Si}, \mathrm{Ca}$, and $\mathrm{Fe}$ (see Fig. 1). The pattern shows that it is the former set of elements that define the initial composition of these stars and not the latter set. Gas is thought to be accreted onto the star from a circumbinary disk, while radiation pressure exerted by the star on the dust grains inhibits the accretion of dust by the star and may also promote a separation of dust and gas in the disk. The dusty disk is betrayed by an infrared excess: HD 44179, also known as the Red Rectangle, is a rather special proto-planetary nebula with a striking infrared excess. On the other hand, BD +39 4926 lacks an infrared excess. Shallow convective envelopes in these extended stars are presumably a key factor in the appearance of their huge abundance anomalies.

Chemical peculiarities of the post-AGB stars are presumably developed from less extreme peculiarities seen in their immediate progenitors, the RV Tauri variables. Discovery of this third category of star displaying the marks of dust-gas winnowing began with the analysis of the RV Tauri variable IW Car (Giridhar et al. 1994). An RV Tauri star is a post-AGB star with an infrared excess (first noted by Gehrz 1972). Subsequent analyses (Giridhar et al. 2005) showed that the effects of dust-gas separation among RV Tauri stars appear limited to the warmer stars $\left(T_{\text {eff }}>4500 \mathrm{~K}\right)$ and to the stars with intrinsic metallicities $[\mathrm{Fe} / \mathrm{H}] \geq-1$. Affected stars have $T_{\text {eff }} \simeq 4500-6500 \mathrm{~K}$ (hotter stars fall outside the instability strip and appear as nonvariable post-AGB stars) and $\log g \simeq$ 0-1. Gonzalez \& Lambert (1997) also discussed the potential importance of the composition of the photosphere (e.g., C/O ratio), and environment (e.g., field vs. globular cluster stars). 
The reasons for these effective temperature and metallicity boundaries are not entirely clear, but the cooler stars possess more extensive convective envelopes that dilute the accreted gas. Dustgas winnowing may be impaired in low-metallicity gas where the dust-to-gas ratio is necessarily lower. The winnowing site is again presumed to be a circumbinary disk; there is increasing evidence that the affected stars are spectroscopic binaries (van Winckel 2007). How the gas is captured onto the star from the circumbinary disk in the presence of a stellar wind remains an unsolved problem, as it does for the post-AGB stars in binaries.

A signature of a star that is affected seriously by dust-gas winnowing is a correlation between an element's abundance and the predicted condensation temperature $T_{\text {cond }}$ (or the abundance in interstellar gas). Estimates of $T_{\text {cond }}$ depend on the initial composition and pressure in a gas and the assumption that cooling of the gas and condensation of grains occurs under equilibrium conditions. Lodders (2003) provides a comprehensive discussion of $T_{\text {cond }}$ estimates for all elements for the solar (O-rich) composition. In the post-AGB stars, and in some Lambda Bootis and RV Tauri stars, the abundance $[\mathrm{X} / \mathrm{H}]$ is smoothly correlated with $T_{\text {cond }}$ (see Fig. 1).

In some Lambda Bootis and RV Tauri stars, $[\mathrm{X} / \mathrm{H}]$ shows no obvious trend with the $T_{\text {cond }}$ (e.g., EQ Cas; Giridhar et al. 2005). Rao \& Reddy (2005) brought order to chaos by noting instead that the $[\mathrm{X} / \mathrm{H}]$ for EQ Cas were correlated with the ionization potential of the neutral atom ("the first ionization potential," or FIP). This raises the intriguing possibility that an alternative or additional process is operating in this one star. The FIP effect is a well-known phenomenon in the solar corona thought to reflect the greater ease with which ions (low FIP elements) rather than neutral atoms (high FIP elements) are fed from the cool chromosphere into the corona. Perhaps EQ Cas is a star where there is a selective feeding of the stellar wind. Thus, in single stars, the stellar wind may control the abundance anomalies, but where the variable is in a binary the circumbinary disk may exert control.

Another possibility for affecting the $[\mathrm{X} / \mathrm{H}]$ versus $T_{\text {cond }}$ correlation (at high $T_{\text {cond }}$ ) is a competition between accretion of metal-free gas and chemical separation due to, e.g., diffusion, gravitational settling, radiative acceleration, or rotational mixing above the convective zone. The diffusion/accretion model for Lambda Bootis stars by Turcotte \& Charbonneau (1993) suggests that timescales for chemical separation can vary by element, thus complicating the abundance pattern established earlier by accretion. The conclusion must be that the dust-gas separation mechanism does not have the same effects on the elemental abundances in the various stars where it appears to operate. Either the mechanism itself, and/or the reaccretion phase of the dust-depleted gas, and/or additional processes that affect chemical separation impact the observed chemical abundance pattern.

In summary, stars with abundance anomalies attributable to dust-gas separation are seen in several parts of the H-R diagram. Associated properties of affected stars, such as age, mass, binarity, or evolutionary status, do not appear to be uniform across the cases. Details in the observed abundance patterns seem to vary between the cases, particularly for elements with high $T_{\text {cond }}$, possibly due to the mechanism itself or additional processes. An accompanying infrared excess may be a warning bell, but it is not a required signature of dust-gas separation. However, to date, the inferred intrinsic metallicities of affected stars are 1/10 solar or greater.

\section{EXAMINATION OF DUST-GAS SEPARATION IN THE ULTRA-METAL-POOR STARS}

As the discussion turns to the ultra-metal-poor stars, we emphasize the importance of the assumptions made in the deter- mination of the $T_{\text {cond }}$ values. Throughout our discussion, the $T_{\text {cond }}$ values are for a solar composition gas. Thus, the values are determined for a gas that is O-rich. One of the ultra-metal-poor stars (HE 0107-5240) is presently severely C-rich, a second ( $\mathrm{HE} 1327-2326$ ) has $\mathrm{C} / \mathrm{O} \simeq 1$, and the $\mathrm{C} / \mathrm{O}$ ratio is unknown for the third (HE 0557-4840). Condensation of grains from C-rich gas provides C-rich solids (e.g., graphite and carbides) and a different set of condensation temperatures.

Calculations by Lodders \& Fegley $(1995,1999)$ have discussed the condensation of grains in $\mathrm{C}$-rich gas. For a gas with $\mathrm{C} / \mathrm{O}>4$, at a pressure representative of a circumstellar region in which dust forms via equilibrium chemistry $\left(P \sim 10^{-7}\right.$ bar $)$, the condensation temperatures $T_{\text {cond }}$ for graphite, $\mathrm{TiC}$, and $\mathrm{SiC}$ (the first three condensates) are approximately 2000, 1560, and $1390 \mathrm{~K}$, respectively. To lower temperatures, the condensation sequence is $\mathrm{Fe}$, $\mathrm{AlN}$, and CaS.

However, the $T_{\text {cond }}$ values are not the whole story. While Ti is effectively removed from the gas at temperatures below its $T_{\text {cond }}$, Si's removal is constrained by the fact that gaseous $\mathrm{SiS}$ is resistant to condensation. The $T_{\text {cond }}$ for graphite but not for $\mathrm{TiC}$ and $\mathrm{SiC}$ declines as $\mathrm{C} / \mathrm{O}$ is decreased to near unity. Condensation temperatures for $\mathrm{Al}, \mathrm{Mg}, \mathrm{Ca}$, and $\mathrm{Fe}$ are all several hundred degrees less than that of Ti. Thus, if the effective $T_{\text {cond }}$ in the grain-forming region is $1500 \mathrm{~K}$ or so, dust-gas separation in a $\mathrm{C}$-rich environment should provide a pronounced Ti deficiency and only for much lower temperatures will deficiencies for other elements be appreciable. Finally, it is possible that dust-gas separation may remove sufficient carbon as graphite to lower the $\mathrm{C} / \mathrm{O}$ ratio toward unity (Lodders \& Fegley 1999). In our figures, we adopt the $T_{\text {cond }}$ values determined for a solar (O-rich) composition gas but discuss possible deviations due to the $\mathrm{C} / \mathrm{O}$ ratio.

\subsection{Has Dust-Gas Separation Modified the Composition of HE 1327-2326?}

Our discussion of HE 1327-2326's composition is based on the abundance analysis by A. Frebel \& N. Christlieb (2007, private communication), which is drawn from new, high signalto-noise ratio Very Large Telescope (VLT) UVES spectra, but confirms and extends previous detailed analyses (Aoki et al. 2006; Frebel et al. 2006). The abundances are plotted as $[\mathrm{X} / \mathrm{H}]$ versus $T_{\text {cond }}$ in Figures 2 and 3 and assume that the star is a subgiant $\left(T_{\text {eff }}=6180 \mathrm{~K}, \log g=3.7\right.$; although our conclusions would be the same, and the following discussion negligibly affected, were we to adopt the abundances assuming the star to be a dwarf). While a classical model atmospheres LTE analysis is performed, predicted corrections are included for effects of stellar granulation. We also show abundances $[\mathrm{X} / \mathrm{H}]$ for two extreme post-AGB stars (HR 4049 and HD 52961), the RV Tauri variables (HP Lyr and UY CMa), and two Lambda Bootis stars (29 Cyg and HD 106223).

There is a resemblance between the compositions of HE 13272326 and the chemically peculiar stars; as $T_{\text {cond }}$ increases, the elemental depletion increases relative to solar. However, HE 1327-2326 is unique because of its extreme $[\mathrm{Mg} / \mathrm{Fe}]$ ratio (see Fig. 2), which is not seen in any of the chemically peculiar stars. Iron appears to be an outlier, since it is the most underabundant element in this star, which is rarely the case for the RV Tauri or Lambda Bootis stars. Notably, S and Zn, important elements of low condensation temperature, have not been detected in HE 1327-2326. The S and Zn upper limits provide no useful constraints on HE 1327-2326, although an abundance estimate for $\mathrm{S}$ may be possible in the future (see below).

Were we to insist that the abundance pattern for the RV Tauri star HP Lyr, with its smooth trend in $[\mathrm{X} / \mathrm{H}]$ versus $T_{\text {cond }}$, be a fair 


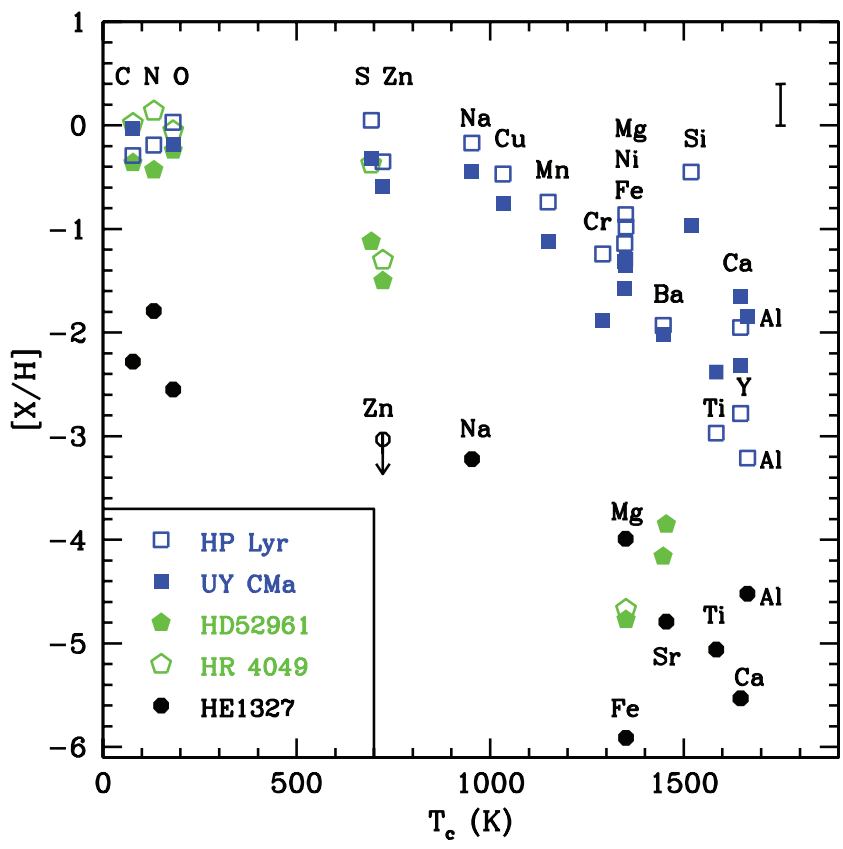

FIG. 2.- $[\mathrm{X} / \mathrm{H}]$ abundances in HE 1327-2326 from A. Frebel \& N. Christlieb (2007, private communication) against the solar condensation temperatures from Lodders (2003). LTE abundances ( filled symbols) and the upper limit for $\mathrm{Zn}$ are shown, all with (3D) corrections for stellar granulation. This abundance pattern is compared to the post-AGB and RV Tauri stars (references in Fig. 1). While the abundance pattern for HE 1327-2326 resembles those of the chemically peculiar stars, the high $[\mathrm{Mg} / \mathrm{Fe}]$ ratio and $\mathrm{Al}$ values are striking. Typical errors are $\leq 0.20 \mathrm{dex}$ shown by the error bar in the upper right; Aoki et al. (2006) suggest slightly larger errors for $\mathrm{C}$ and $\mathrm{N}$ in $\mathrm{HE} 1327-2326$, of 0.24 and 0.30 dex, respectively.

template for testing the suggestion that dust-gas separation has affected HE 1327-2326, we would be bound to note the scatter in Figure 2 for $T_{\text {cond }} \geq 1200 \mathrm{~K}$. In particular, the low abundance of Fe relative to $\mathrm{Al}$ and $\mathrm{Ti}$ in $\mathrm{HE} \mathrm{1327-2326}$ is the opposite in HP Lyr. It seems unlikely that a change in the applied 3D corrections for the (as yet) untested models of stellar granulation can reverse this trend. The $3 \mathrm{D}$ corrections are small for these elements and all of the same sign with an element-to-element scatter of less than 0.2 dex. Estimates of non-LTE corrections assembled by Aoki et al. (2006) for the 1D model also aggravate the situation in that the corrections raise the $\mathrm{Al}$ abundance by 0.4 dex relative to Fe. The non-LTE corrections applicable to the 3D model are unknown.

HP Lyr (and the other RV Tauri comparison star, UY CMa, in Fig. 2) may be an imperfect template for HE 1327-2326. Perhaps, crucially, the $T_{\text {cond }}$ estimates are based on a solar composition and, in particular, on the fact that the Sun is O-rich (i.e., O/C > 1). The measured abundances of $\mathrm{C}$ and $\mathrm{O}$ show that $\mathrm{HE} 1327-2326$ is nominally $\mathrm{C}$-rich now as a subgiant, and most likely the $\mathrm{C} / \mathrm{O}$ ratio has been depressed in evolution to the subgiant branch. (The $\mathrm{C}$ abundance is a mere 0.06 dex greater than the $\mathrm{O}$ abundance, $\mathrm{a}$ difference less than the errors of measurement.) However, an alternative scenario, dust-gas separation in a C-rich environment, also appears to fail to explain the observed composition. As noted above, a signature of such a separation should be an appreciable underabundance of $\mathrm{Ti}$, which is not seen in Figure 2 relative to the other elements.

A comparison to the Lambda Bootis stars is more intriguing. Other than the large $[\mathrm{Mg} / \mathrm{Fe}]$ ratio already noted as peculiar to $\mathrm{HE} 1327-2326$, the relative abundances of $\mathrm{Mg}, \mathrm{Ca}$, and $\mathrm{Ti}$ to each other (all high $T_{\text {cond }}$ elements) are similar to the Lambda Bootis star HD 106223 (see Fig. 3). If the mechanism for dust-

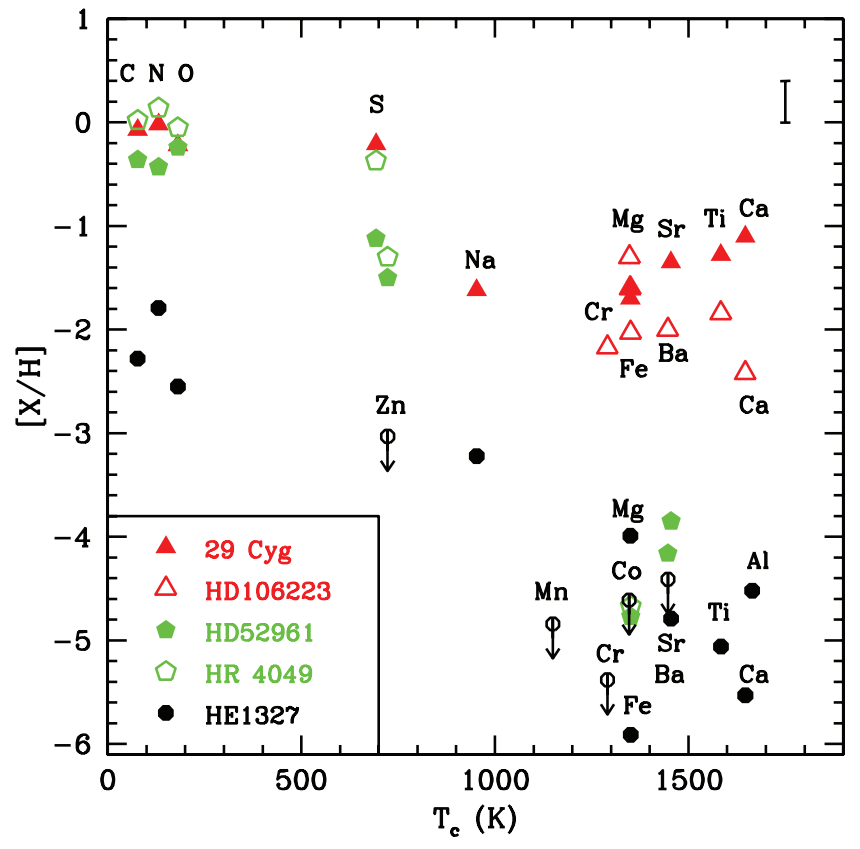

FIG. 3.- $[\mathrm{X} / \mathrm{H}]$ abundances in HE 1327-2326 from A. Frebel \& N. Christlieb (2007, private communication) as in Fig. 2 with additional upper limits noted. The abundance pattern is compared to the post-AGB stars and two Lambda Bootis stars (29 Cyg from Venn \& Lambert 1990 and HD 106223 from Andrievsky et al. 2002).

gas separation is more similar between HE 1327-2326 and the Lambda Bootis stars, possibly complicated by diffusion or another process (see $\S 2$ ), then these stars may be a better comparison template. In Figure 3, both of the Lambda Bootis stars shown have similar depletions of $\mathrm{Ca}, \mathrm{Ti}$, and $\mathrm{Fe}$. Unfortunately, $\mathrm{Al}$ is not available for either Lambda Bootis star. Al, with its very high $T_{\text {cond }}$, is severely depleted in the RV Tauri variables, but less so in HE 1327-2326.

If HE 1327-2326 has undergone dust-gas separation, we can estimate the star's intrinsic metallicity from the $\mathrm{C}, \mathrm{N}$, and $\mathrm{O}$ abundances: an initial metallicity of about -2.0 by the $3 \mathrm{D}$-corrected $\mathrm{C}$, $\mathrm{N}$, and $\mathrm{O}$ abundances but about -1.3 without these corrections. The $3 \mathrm{D}$ corrections are large $(\simeq-0.7 \mathrm{dex})$ for $\mathrm{C}, \mathrm{N}$, and $\mathrm{O}$ because their abundances are derived from molecules $(\mathrm{CH}, \mathrm{NH}$, and $\mathrm{OH})$ whose formation is greatly enhanced by the presence of cooler regions in $3 \mathrm{D}$ models. At an initial metallicity of about -1.3 , the star resembles some post-AGB and RV Tauri stars.

If the initial metallicity of HE 1327-2326 were in fact either $[\mathrm{Fe} / \mathrm{H}]=-2.0$ or -1.3 , this has a small impact on our expectation of the initial abundance ratios for the other elements. For example, $[\mathrm{Ca} / \mathrm{Fe}] \simeq+0.3$ for normal metal-poor stars with $[\mathrm{Fe} / \mathrm{H}]=-2$, and this is approximately seen in Figure 2. A similar ratio can be expected of $\mathrm{Mg}$ and $\mathrm{Ti}$. While $[\mathrm{Ti} / \mathrm{Fe}]$ is slightly larger than expected, the uniquely high $[\mathrm{Mg} / \mathrm{Fe}](\simeq+2)$ ratio is quite out of line with expectations for normal metal-poor stars. Last, in normal metal-poor stars, $[\mathrm{Al} / \mathrm{Fe}]<0$; thus, the high $\mathrm{Al}$ point is also peculiar to HE 1327-2326.

Inspection of the $[\mathrm{X} / \mathrm{H}]$ for HE 1327-2326 (and the other two stars) shows no evidence for a relationship between $[\mathrm{X} / \mathrm{H}]$ and the FIP.

\subsection{Has Dust-Gas Separation Modified the Composition of HE 0107-5240?}

The composition of HE 0107-5240 was determined by Christlieb et al. (2004) and Bessell et al. (2004), who found the star to be a giant with $T_{\text {eff }}=5100 \mathrm{~K}$ and $\log g=2.2$. Their 


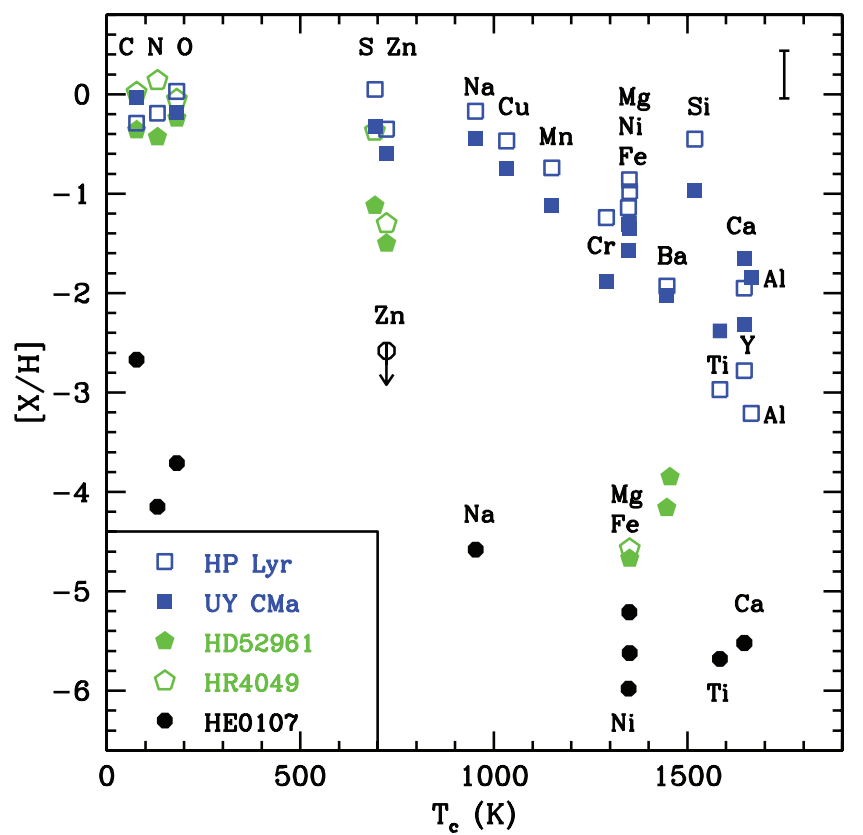

FIG. 4. $-[\mathrm{X} / \mathrm{H}]$ abundances in HE $0107-5240$ from Collet et al. (2006; originally from Christlieb et al. 2004 and Bessell et al. 2004) shown against the solar condensation temperatures from Lodders (2003). LTE abundances ( filled symbols) and the $\mathrm{Zn}$ upper limit are shown, all with (3D) corrections for stellar granulation. This abundance pattern is compared to the post-AGB stars and RV Tauri variables as in Fig. 2. Typical errors for HE $0107-5240$ are $\leq 0.24$ dex, shown by the error bar in the upper right; only the uncertainly on $C$ could be higher, 0.34 dex when determined from the $\mathrm{CH} A-X$ band (Christlieb et al. 2004).

analyses adopted a classical (1D) atmosphere and LTE for the atmosphere and line formation. The derived abundances corrected to those for a 3D atmosphere by Collet et al. (2006) with retention of the assumption of LTE are plotted in Figures 4 and 5.

The abundance pattern for HE 0107-5240 is more similar to the chemically peculiar stars than was HE 1327-2326 discussed above. The $[\mathrm{Mg} / \mathrm{Fe}]$ ratio is similar to that of the chemically peculiar stars, as well as normal metal-poor stars. $\mathrm{Ca}$ and $\mathrm{Ti}$ also show depletions with respect to Fe that are in fair agreement with those of the RV Tauri stars. The upper limits to $\mathrm{Zn}$ and S provide no useful constraint for examining the dust-gas scenario; however, the very low upper limit for $\mathrm{Al}$ is consistent with the predictions.

This star is presently C-rich; $\mathrm{C} / \mathrm{O}$ (by number) is about 20 for the $1 \mathrm{D}$ analysis and about 6 for the $3 \mathrm{D}$ analysis. An initial metallicity of $\simeq-4$ is crudely indicated by the $3 \mathrm{D}$ abundances of $\mathrm{N}$ and $\mathrm{O}$, but $\simeq-2$ by the $1 \mathrm{D} \mathrm{C}$ abundance. The result of the $1 \mathrm{D}$ to $3 \mathrm{D}$ corrections is, as indicated above, a large reduction in the $\mathrm{C}, \mathrm{N}$, and $\mathrm{O}$ abundances, all derived from diatomic molecules $\left(\mathrm{CH}, \mathrm{C}_{2}\right.$, $\mathrm{NH}, \mathrm{CN}$, and $\mathrm{OH}$ ). In $1 \mathrm{D}$, the abundances are larger than those plotted by about $1.2,1.1$, and $0.7 \mathrm{dex}$ for $\mathrm{C}, \mathrm{N}$, and $\mathrm{O}$, respectively. It is an interesting point, possibly one of concern, that $\mathrm{NH}$ and $\mathrm{CN}$ (assuming the $\mathrm{C}$ abundance from $\mathrm{CH}$ and $\mathrm{C}_{2}$ ) give a consistent $\mathrm{N}$ abundance using the $1 \mathrm{D}$ atmosphere but inconsistent abundances (difference is 0.9 dex) using the $3 \mathrm{D}$ atmosphere. The $[\mathrm{C} / \mathrm{O}]$ ratio in HE 0107-5240 is similar to that of the carbon-enhanced metalpoor stars, which also range in metallicity from $[\mathrm{Fe} / \mathrm{H}]=-2$ to -4 (Sivarani et al. 2006).

The appropriateness of using $T_{\text {cond }}$ in Figures 4 and 5 is decidedly questionable due to the star being very $\mathrm{C}$-rich. If the star was and is a single star and its evolution from the main sequence to its present status as a giant even approximately follows expectation, the star must have begun life even more C-rich. Then, if dust-gas separation occurs in either a protostellar, circumstellar,

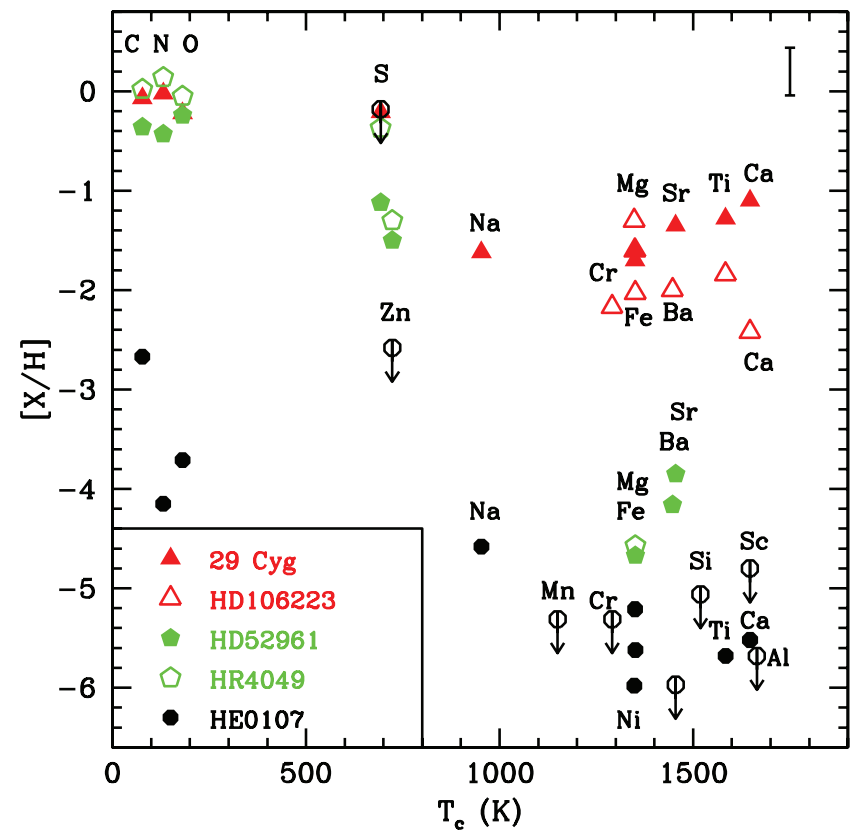

FIG. 5.- $[\mathrm{X} / \mathrm{H}]$ abundances in HE 0107-5420 from Collet et al. (2006) as in Fig. 4 with additional upper limits shown. The abundance pattern is compared to the post-AGB stars and Lambda Bootis stars as in Fig. 3.

or wind environment, it is the $T_{\text {cond }}$ values for C-rich gas that are the relevant quantities. As noted above, a significant deficiency of $\mathrm{Ti}$ is expected for the star accreting substantial amounts of gas. On the other hand, if the star has accreted significant amounts of material from the interstellar medium of the established Galaxy, including the present diffuse interstellar medium, the dust-gas separation was likely to have occurred or is occurring in an O-rich medium, and then Figures 4 and 5 use approximately the appropriate $T_{\text {cond }}$ values. Accretion of interstellar gas that is O-rich requires then an explanation of how HE $0107-5240$ is so C-rich and metal-poor.

\subsection{Has Dust-Gas Separation Modified the Composition of HE 0557-4840?}

The composition is taken from Norris et al. (2007), who determine that the star is a giant and offer spectroscopic and photometric estimates of the effective temperature of 5100 and $4900 \mathrm{~K}$, respectively. The surface gravity is given as $\log g=2.2$. The means of their LTE abundances for a 1D atmosphere are plotted in Figure 6. Corrections for adoption of a 3D model atmosphere are small, except for $\mathrm{C}$ (and the upper limits on $\mathrm{N}$ and $\mathrm{O}$ ), and are plotted in Figure 7. The $\mathrm{C} / \mathrm{O}$ ratio is undetermined for this star.

If the initial composition were that suggested by the $\mathrm{C}$ abundance and $\mathrm{N}$ upper limit for a $1 \mathrm{D}$ atmosphere, the initial $[\mathrm{Fe} / \mathrm{H}] \simeq$ -3 . Taking the $3 \mathrm{D}$ corrections into account, the initial metallicity is likely $\simeq-4$. This lower initial abundance also reduces the scatter in the $[\mathrm{X} / \mathrm{H}]$ values, with a hint that $[\mathrm{X} / \mathrm{H}]$ is independent of $T_{\text {cond. }}$.

There is no pattern in the elemental abundances for HE $0557-$ 4840 that would clearly suggest dust-gas separation. The $[\mathrm{X} / \mathrm{H}]$ values for nearly all elements are flat, in the range of $-4.0>$ $[\mathrm{X} / \mathrm{H}]>-5.5$, and therefore showing similar depletions from a solar composition gas in nearly all elements. This might be unexpected, since the normal metal-poor stars with $[\mathrm{Fe} / \mathrm{H}] \sim-4$ show higher $\alpha$-element abundance ratios, and usually an extremely wide range in the $r$-process elements (McWilliam 1997). However, the $[\alpha / \mathrm{Fe}]$ abundances in HE $0557-4840$ do not fall outside the 


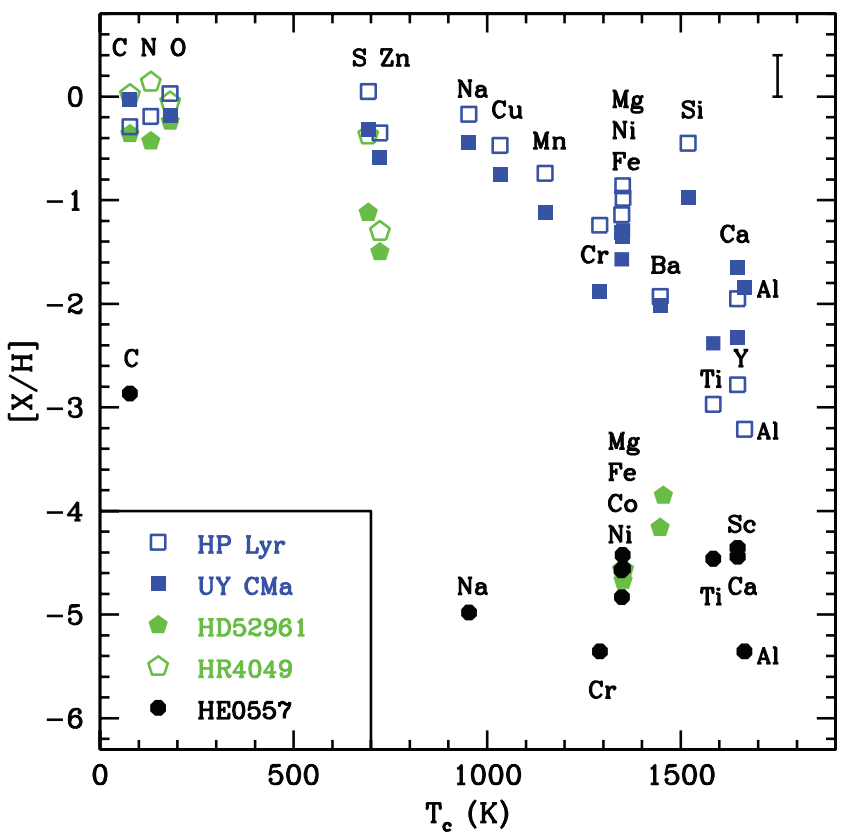

FIG. 6.- $[\mathrm{X} / \mathrm{H}]$ abundances in HE 0557-4840 from Norris et al. (2007) shown against the solar condensation temperatures from Lodders (2003). LTE abundances ( filled symbols) are shown with no corrections for $3 D$ effects. This abundance pattern is compared to the post-AGB stars and RV Tauri variables as in Fig. 2. Typical errors are $\leq 0.20 \mathrm{dex}$, shown by the error bar in the upper right.

range of normal metal-poor star abundances (e.g., $[\mathrm{Mg} / \mathrm{Fe}]$ and $[\mathrm{Ti} / \mathrm{Fe}]$ are within expectations).

\subsection{Other Metal-poor Stars with $[\mathrm{Fe} / \mathrm{H}] \leq-3.5$}

It is natural to ask how ubiquitous the dust-gas separation pattern is among the extremely metal-poor stars with detailed

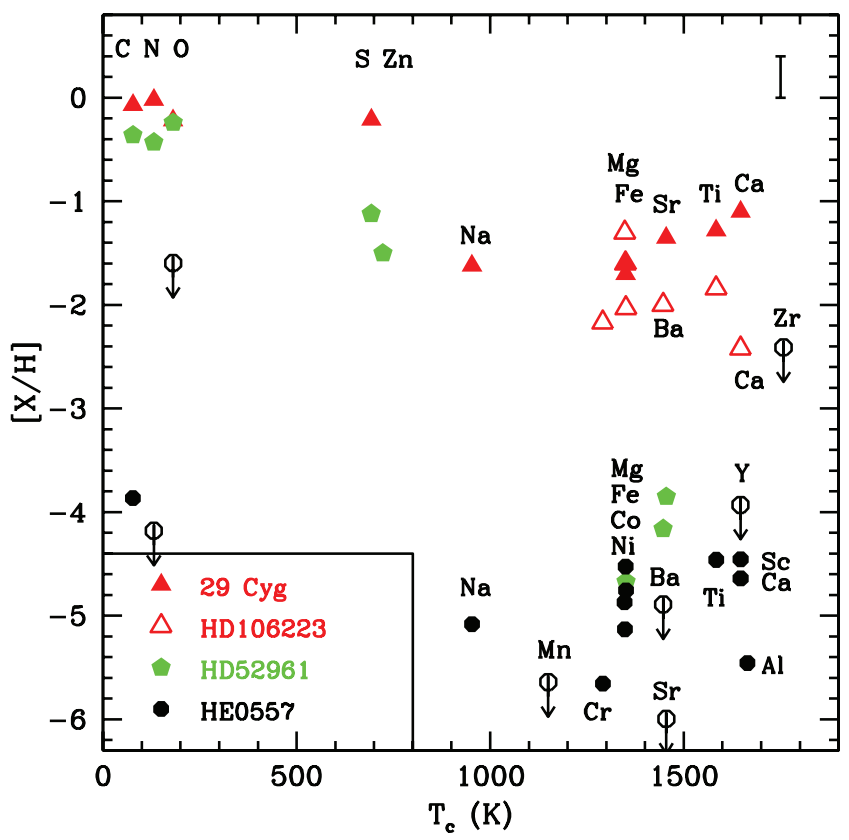

FIG. 7.- $[\mathrm{X} / \mathrm{H}]$ abundances in HE 0557-4840 from Norris et al. (2007) shown against the solar condensation temperatures from Lodders (2003). LTE abundances ( filled symbols) that have been corrected for solar granulation (3D effects) are shown. The 3D corrections have a very small effect on most elements, except carbon. Typical errors are $\leq 0.20$ dex, shown by the error bar in the upper right. The abundance pattern is compared to one of the post AGB stars and the Lambda Bootis stars as in Fig. 3.

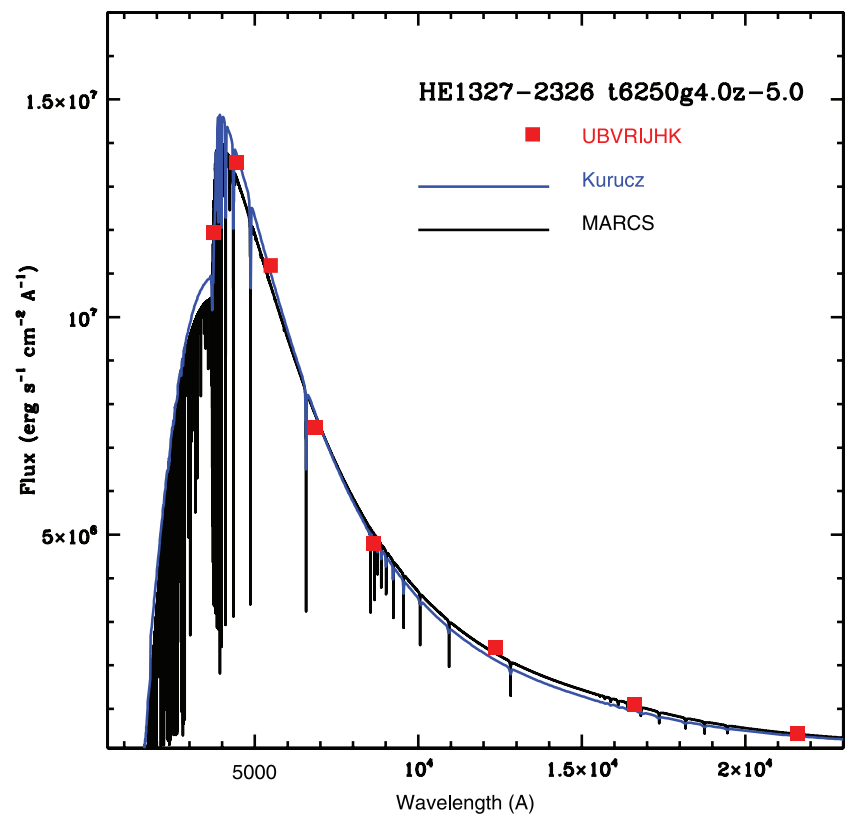

FIG. 8.- Spectral energy distribution for HE 1327-2326 from broadband UBVRI (Aoki et al. 2006) and JHK (2MASS) fluxes. These are compared to model atmosphere flux distributions from both MARCS and Kurucz with $T_{\text {eff }}=6250 \mathrm{~K}$, $\log g=4.0$, and $[\mathrm{Fe} / \mathrm{H}]=-5.0$. Observed magnitudes are scaled to the absolute flux units in order to best fit the RIJ magnitudes. Errors in the fluxes are smaller than the symbol sizes.

elemental abundance determinations. An examination of the literature for stars with $-3.5 \geq[\mathrm{Fe} / \mathrm{H}] \geq-4.0$ shows that none of them shows the dust-gas separation signature. Some examples: CS 29498-043 (Aoki et al. 2004) shows an enhancement in Mg similar to those for $\mathrm{CNO}$, and the $\mathrm{Zn}$ upper limit is significantly lower than that of $\mathrm{CNO}$, which is not predicted in the dust-gas separation pattern. CS 22949-037 (Depagne et al. 2002) also shows an enhancement in $\mathrm{Mg}$ (and $\mathrm{Na}$ ) that is close to that for $\mathrm{CNO}$, and the $\mathrm{Zn}$ determination is significantly lower whereas dust-gas separation would predict a very similar $\mathrm{Zn}$ enhancement to those other elements. Finally, for HE 1300+0157 (Frebel et al. 2007) and HE 1424-0241 (Cohen et al. 2007), several elements ( $\alpha$-elements and iron-group elements) have the same enhancements (or nearly so) as the CNO abundances. Of course, each of these stars has interesting and unusual abundance pattern(s), and while not similar to the predictions for dust-gas separation, they are interesting in terms of the progenitor mass or explosion characteristics of their (small number of) contributing supernovae.

\section{INFRARED EXCESSES}

An association of circumstellar dust, i.e., an infrared excess, with a star that is a candidate for exposure to dust-gas separation strengthens the explanation for the abundance anomalies. The absence of an infrared excess is not fatal to the star's candidacy, as shown by many of the Lambda Bootis stars, and the postAGB star BD +39 4926.

The broadband colors and spectral energy distributions from model atmospheres are shown for the three of the ultra-metalpoor stars in Figures 8-10. JHK magnitudes are from the 2MASS catalog; UBVRI magnitudes are from Aoki et al. (2006) for HE 1327-2326, Christlieb et al.. (2002) for HE 0107-5240, and Beers et al. (2007) for HE 0557-4840. UBVRI magnitudes have been converted to fluxes using the Vega zero points by Colina et al. (1996) and $J H K$ magnitudes converted using the 2MASS Explanatory Supplement ( $\S$ VI.4a). Reddening has been taken 


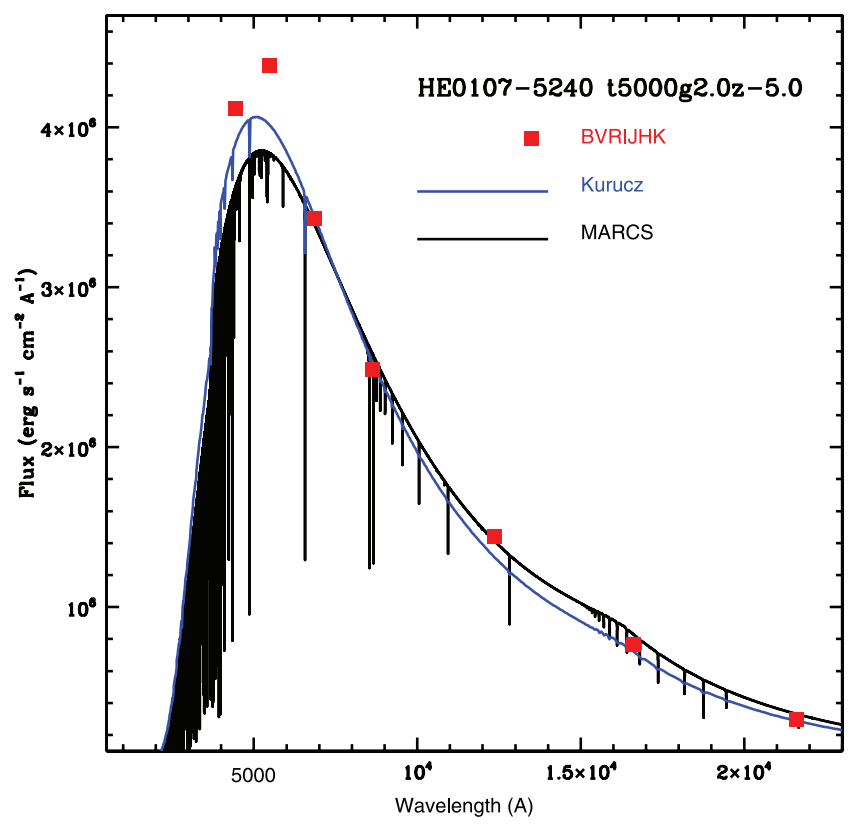

FIG. 9.- Spectral energy distribution for HE 0107-5240. Broadband BVRI colors from Christleib et al. (2002) and JHK (2MASS) colors. The model atmosphere flux distributions are from both MARCS and Kurucz with $T_{\text {eff }}=5000 \mathrm{~K}$, $\log g=2.0$, and $[\mathrm{Fe} / \mathrm{H}]=-5.0$. Observed magnitudes are scaled to the absolute flux units in order to best fit the $R I J$ magnitudes. Errors in the fluxes are smaller than the symbol sizes.

from the reddening maps by Schlegel et al. (1998); $E(B-V)=$ $0.08,0.013$, and 0.04 for HE $1327-2326$, HE $0107-5240$, and HE $0557-4840$, respectively. These were converted to $A_{\lambda}$ based on conversions in Cardelli et al. (1989).

The observed fluxes are compared to model flux distributions from the online grid of available MARCS (Gustafsson et al. 1975; Asplund et al. 1997) and Kurucz models (Castelli \& Kurucz 2004). The closest model parameters to those adopted for each star and available in both grids were $T_{\text {eff }}=6250 \mathrm{~K}, \log g=4.0$, and $[\mathrm{Fe} / \mathrm{H}]=-5.0$ for $\mathrm{HE} 1327-2326$ and $T_{\text {eff }}=5000 \mathrm{~K}, \log g=$ 2.0 , and $[\mathrm{Fe} / \mathrm{H}]=-5.0$ for both HE 0107-5240 and HE 05574840. There are small differences in the models that account for their slight differences in spectral energy distributions; e.g., there are differences in the opacities used, and MARCS models vary stellar mass and $[\alpha / \mathrm{Fe}]$ ratios. The observed fluxes were scaled to overlay the model fluxes best at $R, I$, and $J$; the scale factor is estimated for each star, but would be equivalent to (distance/radius) ${ }^{2}$.

No infrared excess is seen in the colors of any of the ultrametal-poor stars compared to the model flux distributions. While this is an important test for the presence of a dusty disk, it is not definitive. Emission from a disk depends on composition, age, distance from the star, and dust temperature. For many postAGB stars, like HR 4049, the infrared excess is strong in the $K$ band and accompanied by a suppression in the UV continuum (Fig. 1 of Dominik et al. 2003). On the other hand, the circumstellar disk around the post-AGB star HD 56126 (van Winckel \& Reyniers 2000) is detected only beyond $4 \mu \mathrm{m}$, with no excess observed in the $K$ band or below (see Fig. 1 in the review paper by van Winckel 2003). Furthermore, not all Lambda Bootis stars show evidence of their dusty disks either. Paunzen et al. (2003) estimated that $23 \%$ of bona fide Lambda Bootis stars show evidence for circumstellar matter via infrared excesses or Infrared Space Observatory $(I S O)$ and submillimeter $\mathrm{CO}(2-1)$ line emission. In addition, the presence of dust around other A-type stars does not necessarily imply the presence of the Lambda Bootis



FIG. 10.- Spectral energy distribution for HE $0557-4840$ from broadband $B V R$ (Beers et al. 2007) and JHK (2MASS) fluxes. These are compared to model atmosphere flux distributions from both MARCS and Kurucz with $T_{\text {eff }}=5000 \mathrm{~K}$, $\log g=2.0$, and $[\mathrm{Fe} / \mathrm{H}]=-5.0$. Observed magnitudes are scaled to the absolute flux units in order to best fit the $R$ and $J$ magnitudes. Errors in the fluxes are smaller than the symbol sizes.

abundance pattern (Kamp et al. 2002; Dunkin et al. 1997); in the study by Acke \& Waelkens (2004) only one in 24 targets showed a clear Lambda Bootis abundance pattern.

Obviously, the search for an infrared excess should, if possible, be extended to longer wavelengths. Although an infrared excess would clearly identify the presence of a dusty disk, the absence of infrared emission does not preclude the dust-gas separation scenario.

\subsection{Are These Stars Spectroscopic Binaries?}

The presence of a companion seems a prerequisite for efficient dust-gas separation in a post-AGB A-type star and quite probably in the RV Tauri stars. (It is not seemingly a prerequisite for the Lambda Bootis stars.) For this and other reasons, it would be valuable to show whether all three stars are binaries. Presently, the available data are limited on this point.

For HE 0107-5240, Bessell et al. (2004) find no radial velocity variations larger than $0.5 \mathrm{~km} \mathrm{~s}^{-1}$ over $\sim 100$ days (or 373 days when including two spectra taken nearly a year earlier). Similarly for HE 1327-2326, Frebel et al. (2006) find no variations larger than their measurement error or $0.7-1.0 \mathrm{~km} \mathrm{~s}^{-1}$ over $\sim 100$ days (383 if three spectra taken nearly a year earlier are included). For HE $0557-4840$, Norris et al. (2007) find no velocity variations greater than $\pm 0.4 \mathrm{~km} \mathrm{~s}^{-1}$ from observations spanning 40 days. These data do not find evidence for binarity, however. The gold standard for mass transfer across a binary creating abundance anomalies is the study of McClure (1985) of velocity variations for barium stars, which included a $5 \mathrm{yr}$ campaign, with accuracies of $0.5 \mathrm{~km} \mathrm{~s}^{-1}$. An intensive observing campaign may be needed to convincingly test the hypothesis that these ultrametal-poor stars are or are not spectroscopic binaries.

\subsection{The Sulphur Abundance}

For HE 1327-2326, but not for the cooler stars HE 01075240 and HE $0557-5240$, it may be possible to detect $\mathrm{S}_{\mathrm{I}}$ lines 
and determine or at least set an interesting limit on the $\mathrm{S}$ abundance. Observations of Lambda Bootis stars, RV Tauri variables, and A-type post-AGB stars show that S (and Zn) do not share the depletions of iron-group elements (Fig. 1). The upper limit on the $\mathrm{Zn}$ abundance for HE $1327-2326$ is consistent with a smooth interpolation of the $\mathrm{C}, \mathrm{N}, \mathrm{O}$, and $\mathrm{Na}$ abundance.

The strongest $\mathrm{S}_{\mathrm{I}}$ lines of three different multiplets are observed around 10455, 9212, and $8695 \AA$ (Caffau et al. 2005, 2007). Given the atmospheric parameters of HE 1327-2326, we have performed a spectrum synthesis using MOOG (Sneden 1973) and OSMARCS models (Plez 2000). If the star has undergone dust-gas separation, then the intrinsic metallicity as suggested by the $\mathrm{C}, \mathrm{N}$, and $\mathrm{O}$ abundance is $[\mathrm{X} / \mathrm{H}] \simeq-2$ according to the $3 \mathrm{D}$ models but -1.3 for $1 \mathrm{D}$ models. At $[\mathrm{X} / \mathrm{H}]=-2$, the strongest $\mathrm{S}_{\mathrm{I}}$ lines of the 10455,9212 , and $8695 \AA$ multiplets are estimated to have LTE equivalent widths of 15,25 , and $1 \mathrm{~m} \AA$, respectively. NLTE corrections are thought to strengthen the line from these predictions (Takeda et al. 2005). Thus, the 10455 and $9212 \AA$ lines should be detectable; however, the $9212 \AA$ lines are in a region of strong telluric lines.

Detection of the $10455 \AA$ triplet was reported by Nissen et al. (2007). These authors used CRIRES on the VLT to detect with ease the $\mathrm{S}$ I lines in $\mathrm{G} 29-23$, a subdwarf with $[\mathrm{Fe} / \mathrm{H}]=-1.7$. Its atmospheric parameters $\left(T_{\text {eff }}=6200 \mathrm{~K}\right.$ and $\left.\log g=4.0\right)$ are similar to those of $\mathrm{HE} 1327-2326$. Its $[\mathrm{Fe} / \mathrm{H}]$ is within the range supposed for HE 1327-2326 were the $\mathrm{C}, \mathrm{N}$, and $\mathrm{O}$ abundances indicative of the initial composition. The challenge will be to extend the CRIRES observation of G29-23 at $V=10.2$ to HE $1327-$ 2326 at $V=13.5$, a decrease of about a factor of 20 in flux.

\section{CONCLUDING REMARKS}

In this paper, we have outlined the existing evidence for and against the three ultra-metal-poor stars being affected by dustgas separation. The evidence for dust-gas separation having occurred at the stellar photosphere is primarily the abundance pattern, which resembles that of known chemically peculiar stars globally where dust-gas separation has been supported by other observations. The other observations usually include one or more of the following for at least one object within a sample: infrared excesses (usually beyond the $K$ band at $2.2 \mu \mathrm{m}$ ) or other evidence of circumstellar material, evidence of binarity, or the observation that sulfur or zinc do not have the same depletions as the rest of the metals. The latter is a critical test because nondepleted abundances of these two elements are more consistent with dust formation, otherwise requiring random and puzzling variations in nucleosynthetic yields. Most of these observational tests do not yet exist for the ultra-metal-poor stars, or the results are currently inconclusive regarding dust-gas separation.

Searches for radial velocity variations are ongoing in the ultrametal-poor stars to determine whether they are in binary systems. Currently, no variations are found, which suggests that these stars are not binaries, but this evidence cannot be conclusive because of orbit inclinations and/or potentially small amplitude variations. Sulfur and zinc upper limits exist for all three ultra-metal-poor stars; however, these upper limits are presently too high to distinguish between nucleosynthetic origins or dust-gas separation. $K$-band photometry exists for all three ultra-metal-poor stars, and none of them show an infrared excess; however, many RV Tau, post-AGB, and Lambda Boo chemically peculiar stars only show infrared excesses beyond the $K$ band, if at all (many have no detectable infrared excesses). In addition, known chemically peculiar stars suggest that neither dust not binarity are necessary conditions; the Lambda Bootis stars exist as single stars, often without dust; the peculiar post-AGB are binaries, but one lacks dust; the affected RV Tauri stars have an infrared excess, and, although binarity may be a necessary condition, it is as yet unknown observationally that all are binaries. Nevertheless, these observations are possible for the ultra-metal-poor stars, e.g., Spitzer observations at mid-IR wavelengths, and examination of the $\mathrm{S}_{\mathrm{I}}$ lines in near-IR spectroscopy, but have yet to be performed.

One remaining concern is that two of the ultra-metal-poor stars do not occupy atmospheric parameter ranges similar to those of any of the known chemically peculiar stars. HE $0107-$ 5240 and HE 0557-4840 have temperatures like those of the RV Tauri variables, yet higher gravities and lower intrinsic metallicities (if $\mathrm{CNO}$ are the appropriate proxies). Physically, these stars are expected to have atmospheres with deep convective envelopes, and thus, accreted gas from a stellar wind, a circumstellar shell, or a circumbinary disk should be diluted beyond detection. The existence of these envelopes makes it difficult to understand how dust-gas separation effects can be created and sustained in these giants. Indeed, the stars must have received several tenths of a solar mass of separated gas in order that the anomalies be detectable in these giants. Of course, this is a concern for the RV Tauri and post-AGB stars as well. These difficulties are less severe for HE 1327-2326, which is significantly hotter and is expected to have a more shallow convective envelope. HE 1327-2326 occupies (nearly) the same stellar parameter range as the Lambda Bootis stars. Fortunately, detection and analysis of the $\mathrm{S}_{\mathrm{I}}$ lines in this star offers a critical test for any chemical peculiarities due to dust-gas separation.

Stellar astronomers are conditioned to think about stars with very peculiar abundance anomalies - real or imagined. Common sense is often a reliable guide to the boundary between real and imagined. In this regard, if the three stars under consideration here are truly stars of a higher intrinsic metallicity, one might ask why examples have not been seen in well-studied samples of metal-poor stars. The globular clusters spring to mind. Strömgren photometry should be able to pick out those few stars with a reduced metallicity from the rest of the stars showing the monometallicity that is a mark of a globular cluster. Of course, if these stars are due to the random effects of passing through dense interstellar clouds and the effects on the abundances are short lived, then one does not expect to find similar stars in globular clusters, and from objective prism surveys such stars would not stand out as peculiar if the resulting metallicity is greater than $\sim-3$.

It is only because ultra-metal-poor stars are so important as tests of early chemical evolution in the Galaxy that these stars were picked out and studied in detail from high-resolution spectroscopy. It is important that the suspicion be laid to rest that they are not truly ultra-metal-poor but chemically peculiar stars of a more common, if low, metallicity. Then, the focus may be placed exclusively on finding an explanation in terms of stellar nucleosynthesis and the chemical evolution of the young Galaxy (e.g., Iwamoto et al. 2005).

We are grateful to P. Bonifacio for valuable discussions on metal-poor stars and on observations and model calculations of the $\mathrm{S}_{\mathrm{I}}$ lines. We thank Anna Frebel for providing abundances in advance of publication and Katharina Lodders for helpful comments on dust formation in C-rich material. Thanks to Ian Roederer, Inga Kamp, and the anonymous referee for many helpful comments on this manuscript. D. L. L.'s contributions have been supported by the Robert A. Welch Foundation of Houston, Texas. K. A. V. would like to thank NSERC for support through a Discovery grant. 
Acke, B., \& Waelkens, C. 2004, A\&A, 427, 1009

Andrievsky, S. M., et al. 2002, A\&A, 396, 641

Aoki, W., Norris, J. E., Ryan, E. G., Beers, T. C., Christlieb, N., Tsangarides, S., \& Ando, H. 2004, ApJ, 608, 971

Aoki, W., et al. 2006, ApJ, 639, 897

Asplund, M., Gustafsson, B., Kiselman, D., \& Eriksson, K. 1997, A\&A, 318, 521

Aumann, H. H., et al. 1984, ApJ, 278, L23

Baschek, B., \& Searle, L. 1969, ApJ, 155, 537

Beers, T., et al. 2007, ApJS, 168, 128

Bessell, M. S., Christlieb, N., \& Gustafsson, B. 2004, ApJ, 612, L61

Burbidge, E. M., \& Burbidge, G. R. 1956, ApJ, 124, 116

Caffau, E., Bonifacio, P., Faraggiana, R., François, P., Gratton, R. G., \& Barbieri, M. 2005, A\&A, 441, 533

Caffau, E., Faraggiana, R., Bonifacio, P., Ludwig, H.-G., \& Steffen, M. 2007, A\&A, 470, 699

Cardelli, J. A., Clayton, G. C., \& Mathis, J. S. 1989, ApJ, 345, 245

Castelli, R., \& Kurucz, R. L. 2004, in CD-ROM of IAU Symp. 210, Modelling of Stellar Atmospheres, ed. N. Piskunov, W. W. Weiss, \& D. F. Gray (San Francisco: ASP), Poster A20 (astro-ph/0405087)

Christlieb, N., Gustafsson, B., Korn, A. J., Barklem, P. S., Beers, T. C., Bessell, M. S., Karlsson, T., \& Mizuno-Wiedner, M. 2004, ApJ, 603, 708

Christlieb, N., et al. 2002, Nature, 419, 904

Cohen, J., McWilliam, A., Christlieb, N., Shectman, S., Thompson, I., Melendez, J., Wisotzki, L., \& Reimers, D. 2007, ApJ, 659, L161

Colina, L., Bohlin, R., \& Castelli, F. 1996, STScI Instrument Science Rep. CAL/SCS-008 (Baltimore: STScI)

Collet, R., Asplund, M., \& Trampedach, R. 2006, ApJ, 644, L121

Depagne, E., et al. 2002, A\&A, 390, 187

Dominik, C., Dullemond, C. P., Cami, J., \& van Winckel, H. 2003, A\&A, 397, 595

Dunkin, S. K., Barlow, M. J., \& Ryan, S. G. 1997, MNRAS, 286, 604

Frebel, A., Christlieb, N., Norris, J. E., Aoki, W., \& Asplund, M. 2006, ApJ, 638, L17

Frebel, A., Norris, J. E., Aoki, W., Honda, S., Bessell, M. S., Takada-Hidai, M., Beers, T. C., \& Christlieb, N. 2007, ApJ, 658, 534

Frebel, A., et al. 2005, Nature, 434, 871

Gáspár, A., Su, K. Y. L., Rieke, G. H., Balog, Z., Kamp, I., Martínez-Galarza, J. R., \& Stapelfeldt, K. 2008, ApJ, 672, 974

Gehrz, R. D. 1972, ApJ, 178, 715

Giridhar, S., Lambert, D. L., Reddy, B. E., Gonzalez, G., \& Yong, D. 2005, ApJ, 627, 432

Giridhar, S., Rao, N. K., \& Lambert, D. L. 1994, ApJ, 437, 476

Gonzalez, G., \& Lambert, D. L. 1997, AJ, 114, 341

Gray, R. O., \& Corbally, C. J. 1998, AJ, 116, 2530

Gustafsson, B., Bell, R. A., Eriksson, K., \& Nordlund, A. 1975, A\&A, 42, 407

\section{EFERENCES}

Iwamoto, N., Umeda, H., Tominaga, N., Nomoto, K., \& Maeda, K. 2005, Science, 309, 451

Kamp, I., Hempel, M., \& Holweger, H. 2002, A\&A, 388, 978

Kamp, I., \& Paunzen, E. 2002, MNRAS, 335, L45

Lemke, M., \& Venn, K. A. 1996, A\&A, 309, 558

Lodders, K. 2003, ApJ, 591, 1220

Lodders, K., \& Fegley, B., Jr. 1995, Meteoritics, 30, 661

1999, in IAU Symp. 191, Asymptotic Giant Branch Stars, ed. T. Le Bertre, A. Lèbre, \& C. Waelkens (San Francisco: ASP), 279

McClure, R. D. 1985, in Proc. Strasbourg Obs. Colloq. 114, Cool Stars with Excesses of Heavy Elements, ed. M. Jaschek \& P. C. Keenan (Dordrecht: Reidel), 315

McWilliam, A. 1997, ARA\&A, 35, 503

Morgan, W. W., Keenan, P. C., \& Kellman, E. 1943, An Atlas of Stellar Spectra with an Outline of Spectral Classification (Chicago: Univ. Chicago Press)

Nissen, P. E., Akerman, C., Asplund, M., Fabbian, D., Kerber, F., Kaufl, H. U., \& Pettini, M. 2007, A\&A, 469, 319

Norris, J. E., Christlieb, N., Korn, A. J., Eriksson, K., Bessel, M. S., Beers, T. C., Wisotzki, L., \& Reimers, D. 2007, ApJ, 670, 774

Paunzen, E., Kamp, I., Weiss, W. W., \& Wiesemeyer, H. 2003, A\&A, 404, 579

Plez, B. 2000, in IAU Symp. 177, The Carbon Star Phenomenon, ed. R. F. Wing (Dordrecht: Kluwer), 71

Rao, N. K., \& Reddy, B. E. 2005, MNRAS, 357, 235

Savage, B., \& Sembach, K. 1996, ApJ, 470, 893

Schlegel, D. J., Finkbeiner, D. P., \& Davis, M. 1998, ApJ, 500, 525

Sivarani, T., et al. 2006, A\&A, 459, 125

Sneden, C. 1973, ApJ, 184, 839

Su, K. Y. L., et al. 2005, ApJ, 628, 487

Suda, T., Aikawa, M., Machida, M. N., Fujimoto, M. Y., \& Iben, I., Jr. 2004, ApJ, 611, 476

Takeda, Y., Hashimoto, O., Taguchi, H., Yoshioka, K., Takada-Hidai, M., Saito, Y., \& Honda, S. 2005, PASJ, 57, 751

Takeda, Y., et al. 2002, PASJ, 54, 765

Tominaga, N., Umeda, H., \& Nomoto, K. 2007, ApJ, 660, 516

Tumlinson, J. 2007a, ApJ, 664, L63 2007b, ApJ, 665, 1361

Turcotte, S., \& Charbonneau, P. 1993, ApJ, 413, 376

Umeda, H., \& Nomoto, K. 2003, Nature, 422, 871

van Winckel, H. 2003, ARA\&A, 41, 391 2007, Baltic Astron., 16, 112

van Winckel, H., Mathis, J. S., \& Waelkens, C. 1992, Nature, 356, 500

van Winckel, H., \& Reyniers, M. 2000, A\&A, 354, 135

van Winckel, H., Waelkens, C., \& Waters, L. B. F. M. 1995, A\&A, 293, L25

Venn, K. A., \& Lambert, D. L. 1990, ApJ, 363, 234

Waelkens, C., van Winckel, H., Bogaert, E., \& Trams, N. R. 1991, A\&A, 251, 495 